\title{
Karyotypic Variation in Ten Strains of Indian Raddish (Raphanus sativus L.)
}

\author{
Pulak Mukherjee \\ University of Calcutta, India
}

Received August 23, 1977

Raphanus is an important genus in the family Cruciferae belonging to the tribe Brassiceae. A considerable amount of work involves both intergeneric and interspecific crosses have been carried out in this one of the important horticultural crops (Karpechenko 1922, 1924, Nishiyama 1949, 1952, Sato 1955 and Sampson 1962). Though a good amount of work has been carried out in this genus, a detailed study of karyotype with the aid of improved methods as well as the meiotic segregation has not yet been carried out. As a large number of strains have been raised in cultivation in India, it is specially necessary in Raphanus sativus L. The present paper deals with the karyomorphological studies of ten different horticultural strains of Raphanus sativus L. with the help of improved techniques in order to understand the interstrain difference and the role of strucutral alterations in the evolution of different cultivated strains of Raphanus sativus L.

\section{Materials and methods}

The materials of the present investigation include nine different horticultural strains of Raphanus sativus L., seven purchased from "Calcutta Seed Suppliers" and two local cultivars of Arambagh, Hooghly District and one wild type collected from Eastern Himalayan region. The following strains have been studied.

Raphanus sativus L. strain "Deshi red long", $R$. sativus L. strain "Deshi purple long", $R$. sativus L. strain "Sutton's long white", $R$. sativus L. strain "Sutton's long scarlet", $R$. sativus L. strain "Sutton's scarlet globe", $R$. sativus $\mathrm{L}$. strain "Sutton's Crimson French breakfast", $R$. sativus L. strain "Sutton's Chinese white", $R$. sativus L. strain "Sutton's Contai long", $R$. sativus L. strain "Sutton's Contai short", $R$. sativus (Wild) collected from Darjeeling.

For somatic study the seeds were germinated on moist filter paper and healthy root tips were selected and pretreated with aq. aesculine (Sharma and Sarkar 1955) for 2 hours $30 \mathrm{~min}$. at $8^{\circ} \mathrm{C}$. Next propiono orcein staining technique was followed after fixing the root-tips in propionic alcohol $1: 2$ for $30 \mathrm{~min}$. For meiotic study, propiono carmine staining technique was followed fixing the flower buds in $1: 2$ propionic alcohol for 24 hours and storing them in $70 \%$ ethyl alcohol.

1 Present address: Rice Research Institute, P.O. Chinsurah, Dist. Hooghly, West Bengal, India. 


\section{Observations}

Studies on the chromosomes of nine different strains of Raphanus sativus show
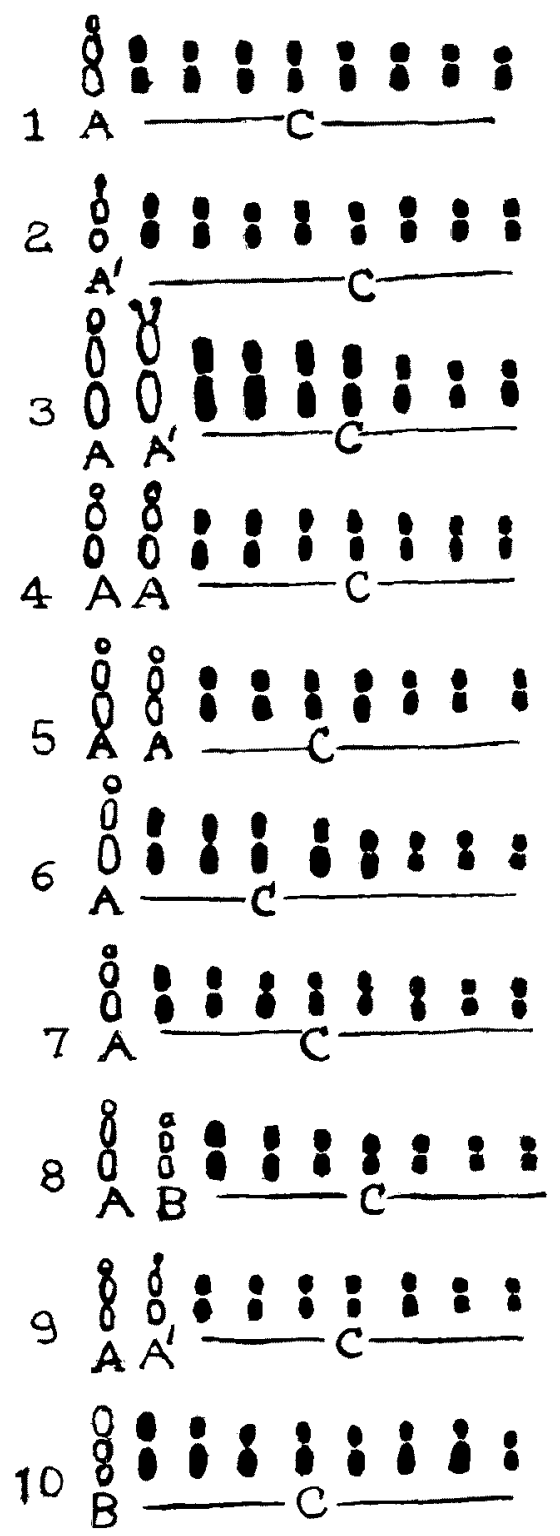

Figs. 1-10. Idiograms of the strains Sutton's long white; Deshi purple long; Deshi red long; Sutton's scarlet globe; Sutton's Crimson French breakfast; Sutton's Chinese white; Contai long; Contai short; and Sutton's long scarlet and $R$. sativus (wild) respectively showing $2 n=18$ chromosomes. a good deal of similarity in their chromosome number, size and morphology. All of them contain $2 \mathrm{n}=18$ chromosomes in their complements. The chromosome size varies from $4 \mu$ to $1.1 \mu$. On the basis of their size and position of the primary and secondary constrictions, a number of chromosome types is seem to be common to all of them, though they differ from one another in the minute details of the karyotype. A general description of the representatives of the type is given below and their details will be discussed under different varieties.

Type A - A long to medium sized chromosome with two constrictions, primary and secondary, one nearly median and the other subterminal at the distal end of one segment.

Type $\mathrm{A}^{\prime}-\mathrm{A}$ comparatively long to medium sized chromosome with median primary constriction and with a satellite at the distal end of one of the arms occasionally the satellite is splitted.

Type B - Medium sized chromosome with two constrictions, primary and secondary, one nearly submedian in position and the other located in the middle of the longer arm dividing the chromosome into three equal segments.

Type $\mathrm{C}$-Comparatively long to medium sized chromosome with median to submedian primary constriction.

The following strains have different numbers of the above mentioned types.

1. Raphanus sativus L. strain "Sutton's long white"

$$
2 \mathrm{n}=18=\mathrm{A}_{2}+\mathrm{C}_{16}=2.3 \mu-1.6 \mu
$$

(Fig. 1, Photo 1)

2. R. sativus L. strain "Deshi purple long" 


$$
2 \mathrm{n}=18=\mathrm{A}_{2}^{\prime}+\mathrm{C}_{18}=2.2 \mu-1.7 \mu \text { (Fig. 2). }
$$

Meiotic analysis shows nine bivalents in metaphase.

3. R. sativus L. strain "Deshi red long"

$$
2 \mathrm{n}=18=\mathrm{A}_{2}+\mathrm{A}_{2}^{\prime}+\mathrm{C}_{14}=4 \mu-1.3 \mu \text { (Fig. 3). }
$$

Meiotic shows nine bivalents in metaphase I.

4. R. sativus L. strain "Sutton's scarlet globe"

$$
2 \mathrm{n}=18=\mathrm{A}_{4}+\mathrm{C}_{14}=2.7 \mu-1.3 \mu \text { (Fig. 4). }
$$

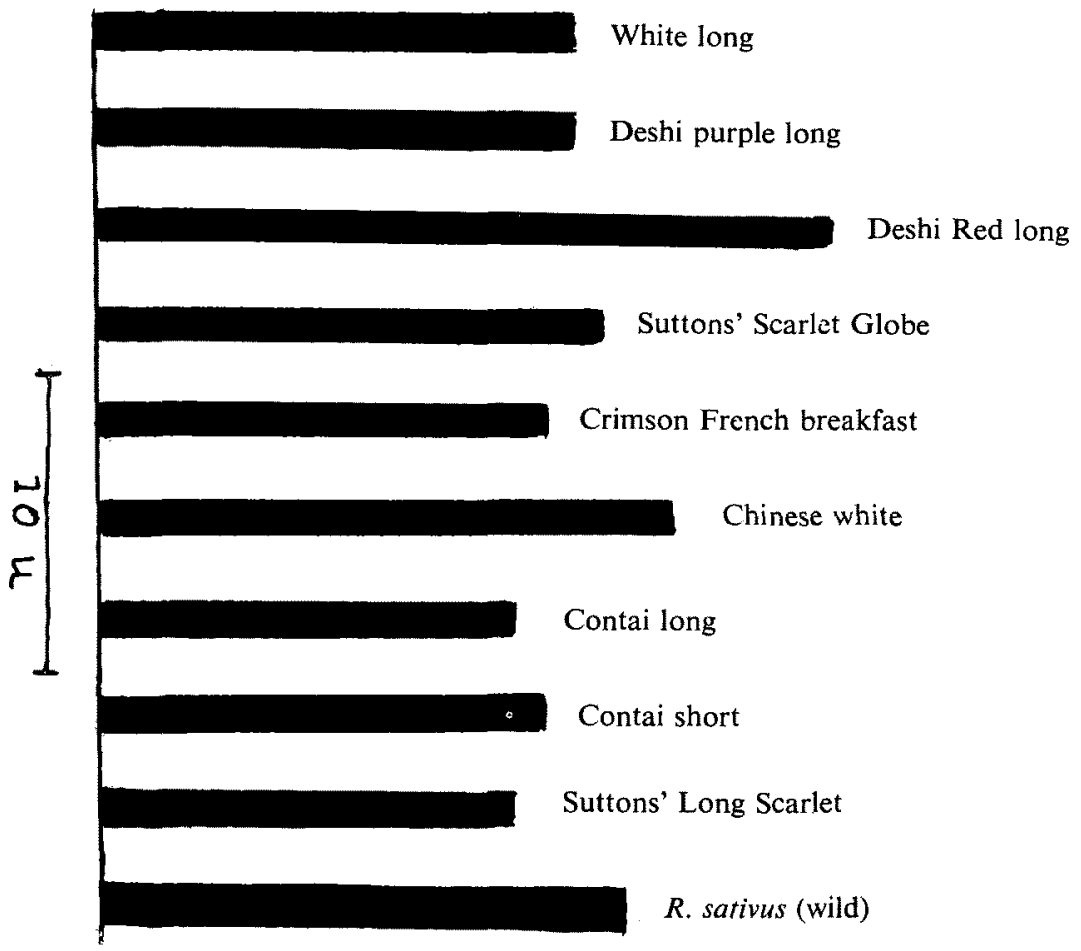

Fig. 11. Histogram showing total amount of chromatin matter in length of the haploid complements of the different varieties of $R$. sativus.

5. R. sativus L. strain "Sutton's crimson French breakfast"

$$
2 \mathrm{n}=18 \mathrm{~A}_{4}+\mathrm{C}_{14}=2.5 \mu-1.1 \mu \text { (Fig. 5) }
$$

6. R. sativus $\mathrm{L}$. strain "Sutton's Chinese white"

$$
2 \mathrm{n}=18=\mathrm{A}_{2}+\mathrm{C}_{16}=3.6 \mu-2 \mu \text { (Fig. 6). }
$$

Meiosis shows nine chromosome in metaphase II.

7. R. sativus L. strain "Contai long"

$$
2 \mathrm{n}=18=\mathrm{A}_{2}+\mathrm{C}_{18}=2.8 \mu-1.6 \mu \text { (Fig. 7) }
$$

8. R. savtius $\mathrm{L}$. strain "Contai short"

$$
2 \mathrm{n}=18=\mathrm{A}_{2}+\mathrm{B}_{2}+\mathrm{C}_{14}=3 \mu-1.7 \mu \text { (Fig. 8). }
$$

9. $R$. sativus $\mathrm{L}$. strain "Sutton's long scarlet"

$$
2 \mathrm{n}=18=\mathrm{A}_{2}+\mathrm{A}_{2}{ }_{2}+\mathrm{C}_{14}=2.6 \mu-1.3 \mu \text { (Fig. 9). }
$$

10. R. sativus $\mathrm{L}$. (Wild collected from Darjeeling).

$$
2 \mathrm{n}=18=\mathrm{B}_{2}+\mathrm{C}_{16}=2.3 \mu-1 \mu \text { (Figs. } 10,12 \mathrm{~b} \text { ) }
$$


Meiotic analysis shows nine bivalents in diakinesis and anaphase is normal (Fig. 12a).

\section{Discussion}

The chromosome number of $R$. sativus is observed as $2 n=18$ confirming the previous report by Karpechenko (1924), Sato (1955) and Samson (1962). Although ten varieties have been studied, a general homogeneity in the karyotype has been noted. The strains also have been found to differ with respect to minute strutural details of the karyotype. The general similarity in chromosome morphology, consisting mainly of medium sized chromosomes which are mostly medianly constricted and with one or two pairs of secondary constrictions, indi-
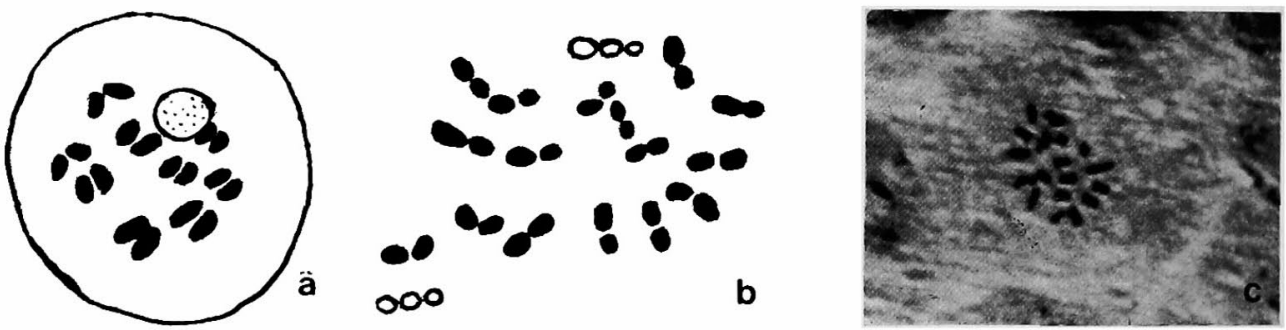

Fig. 12. a-c. a, diakinesis of $R$. sativus (wild) showing $\mathrm{n}=9$ chromosomes. b, somatic plate of $R$, sativus (wild) showing $2 \mathrm{n}=18$ chromosmes. c, metaphase plate.

cates that all the different strains are allied to each other. However, regarding the basic number in the karyotype, the strains differ with respect to minute details in chromosome morphology. These facts indicate that even at an intraspecific level minute alteration of chromosomes have been associated in the origin of new strains.

Although structural alterations have played an important role in the evolution of strains, it has been shown that meiosis has been found to be regular in all cases. This fact indicates that whatever may have been the alteration, extensive cultivation, accompanied by selection has resulted the stabilization of the homozygous forms. Such structural alteration in the origin of the strains and their gradual homozygosity have been reported in the species of Sorghum (Sharma and Bhattacharyya 1957), Hordeum (Sharma 1956, Sharma and Mukherjee 1956), Secale (Bose 1956, 1957) and in Brassica (Mukherjee 1974, 1975, 1977).

However, in one of the strains, viz. strain "Deshi red long" the chromosome size has been found to be comparatively longer than the rest as noted from the histogram as well (Fig. 11). It is likely that this strain is the representative of the primitive stage and that other strains with shorter chromosomes are evolved through horticultural practices. In that case the diminution in chromosome size is assumed to have taken place during evolution. Examples of such diminution of chromosome size are prevalent in higher plants (Stebbins 1951, Sharma and Sharma 1959). Another strain of $R$. sativus was collected in wild condition in the Himalayas (Eastern Himalayas region, altitude $1000^{\prime} \mathrm{ft}$.) and shows $\mathrm{n}=9$ bivalents. The occurrence of this wild species of Raphanus is quite remarkable. 


\section{Summary}

The karyomorphological studies of the ten different strains of $R$. sativus show homogeneity in their gross morphology. However, minute structural difference between different strains are noticed in relation to the number of secondary constriction, the position of the primary constrictions as well as in the arm ratio. However, in one of the strains, viz. "Deshi red long" the chromosome size has been found to be comparatively longer than the rest as noted from the histogram as well. It is likely that this strain is the representative of the primitive stage and that other strains with shorter chromosomes are evolved through horticultural practices. In that case the diminution in chromosome size is assumed to have taken place during evolution. It has been suggested that the structural changes in chromosome have played an important role in the evolution of different taxonomic units of Raphanus sativus L.

\section{Acknowledgement}

The author wishes to express his deep gratitude and indebtedness to Prof. A.K. Sharma, D.Sc., FNA, Head of the Department of Botany, University of Calcutta for his guidance and facilities provided. The author is also grateful to Prof. (Mrs.) A. Sharma, Ph. D., D. Sc., Department of Botany, University of Calcutta for her invaluable help and advice at different stages of the work. This research has been financed in a part by a grant made by the U.S. Department of Agriculture under PL 480 Project No. FG-IN-318.

\section{References}

Bose, S. 1956. Aberrations in the nucleolar chromosome of inbred rye. Hereditas 42: 263-291.

-1957. Aberrations in the nucleolar chromosome of inbred rye II. Size variation in inbred lines and population plants. Hereditas 43: 621-643.

Karpechenko, G.D. 1922. The number of chromosomes and the genetic correlation of cultivated Cruciferae. Bull. Appl. Bot. and Pl. Breed. 2: 3-14.

-1924. Hybrids of Raphanus sativus L. $\times$ Brassica oleracea L. J. Genet. 14: 375-395.

Mukherjee, P. 1974. Inter-strain difference in karyotype of Brassica oleracea L. Curr. Sci. 43 (18): $592-594$.

-1975. Karyomorphological studies of ten strains of Indian mustard (Brassica juncea Coss.). Euphytica 24: $483-486$.

-1977. The structural and numerical alterations of chromosomes in Brassica campestris L. Cytologia 42: 181-188.

Nishiyama, I. 1949. Studies on artificial polyploid plants. Artificial development of seeds in reciprocal crosses between diploid and tetraploid Raphanus. The Res. Food Sci. Kyoto Univ. No. 2: 49-54.

-1952. Polyploid studies in Brassiceae I. The seed development in reciprocal crosses between diploid and tetraploid Raphanus. The Res. Food Sci. Kyoto Univ. No. 3: 1-7.

Sato, Z. 1955. Induction of polyploidy and mechanism of chromosome doubling by aurantia. Jap. J. Gent. 30: 12-16.

Sampson, D.R. 1962. Inheritance of pollen stigma incompatibility in the Cruciferae. Canad. J. Genet. Cyt. 4: 38-49.

Sharma, A.K. 1956. Chromosome studies in some Indian barley. Proc. Nat. Inst. Sci. 22: 246- 
256.

-and Sarkar, S.K. 1955. A new technique for the study of chromosome of palms. Nature 76: 261.

-and Mukherjee, R.N. 1956. Chromosome studies in some Indian barley II. Proc. Inst. Acad. Sci. 43: 279-287.

-and Bhattacharyya, D. 1957. Chromosome studies in Sorghum. Cytologia 22: 289-311. -and Sharma, A. 1959. Chromosomal alteration in relation to speciation. Bot. Rev. 25: 514 544.

Stebbins, G.L. 1950. Varition and Evolution in Plants. Columbia Univ. Press, New York. 